\title{
Relations of Mood with Body Mass Index Changes in Severely Obese Women Enrolled in a Supported Physical Activity Treatment
}

\author{
James J. Annesi
}

YMCA of Metropolitan Atlanta, Atlanta, GA, USA

\section{Key Words}

Mood · Physical activity · Weight loss · Psychological aspects

\section{Summary}

Background/Aims: It has been suggested that physical activity may affect weight reduction outcomes through associated improvements in mood. Relations of physical activity, mood, and weight change are not well understood in persons classified as severely obese (BMI $\geq 40$ $\left.\mathrm{kg} / \mathrm{m}^{2}\right)$, however. This research tested these relationships in women with severe obesity. Methods: 57 women with a mean BMI of $43.8 \mathrm{~kg} / \mathrm{m}^{2}$ were enrolled in a cognitivebehavioral exercise support treatment with group-based nutrition information. Measurement of depression, tension, overall mood, and BMI was taken at baseline and month 6, and exercise session attendance was recorded. Results: The treatment was associated with significant improvements in depression, tension and total mood disturbance scores as well as in BMI over 6 months. Changes in mood scores that were more positive were correlated with a greater reduction in BMI. Mean attendance in the prescribed 3 session/week exercise regimen was $46.0 \%$, and attendance was significantly correlated with changes in tension and total mood disturbance scores, and approached significance with changes in depression scores. Conclusion: Findings suggested significant relations of mood and weight changeas well as of physical activity and mood in severely obese women associated with a treatment of moderate physical activity. With extensions of this research, weight loss theory and treatment may benefit.

\section{Introduction}

In the USA $33.4 \%$ of women meet the present classification of obesity (BMI $\geq 30.0 \mathrm{~kg} / \mathrm{m}^{2}$ ) [1]. Approximately $6.9 \%$ of women fulfill the criteria for severe, morbid, extreme, or class III obesity [1], which is defined as a BMI of $40.0 \mathrm{~kg} / \mathrm{m}^{2}$ or greater. Based on degree of overweight, these individuals are at a progressively increased risk for type 2 diabetes, hypertension, coronary heart disease, various cancers, and premature death [2]. Because diets of severe caloric restrictions overall have been ineffective [3], more invasive methods such as pharmacotherapy and bariatric surgery have been suggested to reduce weight [4].

Although physical activity has been associated with only moderate amounts of additional weight loss over the short term when added to a modified eating plan [5], it is the strongest predictor of maintained weight loss [6]. In fact, it is rare for persons who have lost a considerable amount of weight to maintain the loss without a program of regular exercise [7]. As it has been shown that the association between physical activity and weight loss is not completely explained by caloric expenditure, additional mechanisms have been considered [8]. For example, the association of even minimal amounts of physical activity with both acute and chronic improvements in mood [9] has been suggested as playing a part in explaining weight loss [10]. Obesity is associated with mood disorders in females [11,12], and associations of depressive symptoms with binge eating disorder and obesity have been identified [13]. An association between physical activity, overall mood, and weight loss was suggested in a large sample of those who attained and maintained substantial reductions in weight [7]. This research, however, was cross-sectional, and thus could not imply causality.

A number of different explanations concerning the interrelations of exercise, mood, and overweight are worthy of consid-

James J. Annesi, Ph.D.

YMCA of Metropolitan Atlanta

100 Edgewood Avenue, NE, Suite 1100

Atlanta, Georgia 30303 , USA

Tel. +1 404-267 5355, Fax -527 7693

E-mail jamesa@ymcaatlanta.org 
eration. For example, biological research suggests significant relations between overweight, depression, and metabolic syndrome in women [14]. Baker and Brownell [15], however, adopted a behavioral perspective based on social cognitive theory [16] and proposed a model suggesting that participation in a physical activity program is associated with improved mood and a better 'psychological climate' which, in turn, is associated with weight loss. Social cognitive theory posits a triadic, reciprocal relationship between person, environment and behavior, and suggests that persons will receive reinforcement value from connecting behaviors (e.g. physical activity) with valued outcomes (e.g. improved mood). Self-efficacy is a construct embedded in social cognitive theory that is a belief that one has the ability to be successful at specific behaviors (e.g. those associated with weight loss) [17]. In summarizing effects emanating from a regimen of physical activity, Baker and Brownell stated that 'positive changes in overall well-being, and in specific variables such as depression and anxiety, could lead to a healthier psychological climate in which individuals have more cognitive and emotional resources, as well as motivation and energy, to sustain the long-term commitment to a weight-loss program' [15, p 320].

In preliminary testing of the Baker and Brownell model, several embedded propositions were supported [18]. For example, physical activity was significantly associated with changes in measures of self-efficacy, anxiety, and depression; and changes in mood scores were significantly correlated with changes in body composition. Within the experimental protocol used, exercise types and intensities were largely left to participants' preferences, and application of an evidence-based exercise support treatment (The Coach Approach) was associated with greater adherence than a control condition [18]. Severely obese individuals who may find exercise especially uncomfortable, and may thus respond differently on psychological factors were not, however, specifically studied. Thus, generalizability of findings to this group was unknown.

To provide data on psychological correlates of weight loss and how severely obese women respond to a treatment where manageable amounts of physical activity rather than caloric restriction is emphasized, this investigation was initiated. Specifically, the following research questions were posed:

i) Was the physical activity and nutrition information treatment associated with significant improvements in depression, tension, and total mood disturbance scores as well as in BMI over 6 months?

ii) Did depression, tension, and total mood disturbance scores at baseline significantly correlate with BMI change?

iii) Did changes in depression, tension, and total mood disturbance scores over 6 months significantly correlate with BMI change?

iv) Was exercise session attendance significantly correlated with changes in depression, tension, and total mood disturbance scores?

\section{Material and Methods}

\section{Subjects}

Women responded to solicitations for volunteers in local newspapers. Inclusion criteria for this investigation was i) age of 21 to 65 years, ii) BMI $\geq 40 \mathrm{~kg} / \mathrm{m}^{2}$, iii) no regular exercise ( $\leq 1 \mathrm{session} /$ week) within the previous year, iv) a reported goal of weight loss, and v) reported failures with caloric restriction diets. Exclusion criteria were i) inadequate physical condition for exercise, ii) pregnant or planning to become pregnant soon, and iii) taking medication for weight loss or a psychiatric disorder. A written statement of adequate health to participate was required from a physician. Appropriate institutional review board approval and written consent from all subjects was received. The age range of the 57 subjects was $21-52$ years (mean $\pm \mathrm{SD}=44.4 \pm 10.3$ years). Approximately $93 \%$ of the sample was in the lower-middle to middle classes. The ethnic make-up was 49\% White, $42 \%$ African American, and 9\% of other ethnicities. BMI was $40.15-49.88 \mathrm{~kg} / \mathrm{m}^{2}\left(\right.$ mean $\left.\pm \mathrm{SD}=43.75 \pm 2.89 \mathrm{~kg} / \mathrm{m}^{2}\right)$

\section{Measures}

Consistent with previous research [19], exercise session attendance was the ratio of sessions attended divided by the 'optimal' number of sessions of 72 ( 3 sessions assigned per week $\times 24$ weeks), expressed as a percentage. Exercise sessions completed were recorded electronically through a computer system that was suggested valid through strong significant correlations $(r$-values $=0.42-0.55)$ with changes in measures of cardiorespiratory function (e.g. $\mathrm{VO}_{2} \max$, blood pressure, resting heart rate) [20].

The depression and tension scales of the Profile of Mood States Short Form [21] required responses to five 1- to 3-word items anchored by 0 (not at all) and 4 (extremely), which reflected on feelings occurring, 'during the past week including today'. The Total Mood Disturbance scale is an aggregate measure of negative mood derived by summing the Profile of Mood States scales scores of depression, tension, fatigue, confusion, and anger, and subtracting the positive scale score of vigor. Internal consistency ranged from 0.84 to 0.95 , and test-retest reliability at 3 weeks averaged 0.69 [21]. The depression scale had a correlation of 0.61 with the Beck Depression Inventory, and the tension scale had a correlation of 0.80 with the Manifest Anxiety Scale [21].

\section{Procedure}

Subjects were provided no-cost access to YMCA exercise centers. A series of six 1-hour meetings with a credentialed exercise specialist was provided where a cognitive-behavioral exercise support protocol (The Coach Approach) [16] was administered over 6 months. A supporting computer program adjusted treatment components based on responses to validated surveys. Methods included instruction on an array of self-management and self-regulation skills such as goal setting, cognitive restructuring, and stimulus control; exercise types based on individual preferences (e.g. walking, water-based exercise), with adjustments of intensities and durations to induce positive post-exercise feeling changes, and instructor-led group exercise to promote feelings of social cohesion. Exercise plans were assigned for 3 sessions/week. The Coach Approach protocol was intended to increase feelings of mastery, competence and self-efficacy, and to maximize adherence [22].

Because it is the current standard in behavioral weight loss treatment, information in nutritional practices were included. Registered dietitians provided 6 biweekly, 1-hour nutrition information sessions in small groups. The standardized protocol used (i.e. Cultivating Health) included curriculum components of understanding calories, carbohydrates, protein, and fats; calculating caloric needs; using the food pyramid; developing a plan for appropriate snacking; and menu planning [23]. No specific caloric or fat restrictions were imposed.

Assessments were administered in a private area at baseline and week 24 . All applicable institutional and governmental regulations concerning the ethical use of human volunteers were followed during this research. 
Table 1. Within-group changes over 6 months in depression, tension, and total mood disturbance scores, and BMI $(\mathrm{n}=57)$

\begin{tabular}{|c|c|c|c|c|c|c|}
\hline & \multicolumn{2}{|c|}{ Baseline } & \multicolumn{2}{|c|}{ Month 6} & \multirow[t]{2}{*}{$t$} & \multirow[t]{2}{*}{$d^{\mathrm{b}}$} \\
\hline & Mean & $S D$ & Mean & $S D$ & & \\
\hline Depression $^{\mathrm{a}}$ & 3.07 & 2.26 & 2.62 & 1.91 & $2.03 *$ & 0.20 \\
\hline Tension $^{\mathrm{a}}$ & 4.16 & 3.93 & 2.49 & 2.10 & $3.71^{*}$ & 0.42 \\
\hline $\begin{array}{l}\text { Total mood } \\
\text { disturbance }^{\mathrm{a}}\end{array}$ & 14.28 & 15.20 & 5.22 & 11.28 & $4.39 *$ & 0.60 \\
\hline BMI & 43.75 & 2.89 & 42.47 & 3.99 & $3.45^{*}$ & 0.44 \\
\hline
\end{tabular}

${ }^{a}$ Depression, tension, and total mood disturbance are scales of the Profile of Mood States Short Form.

${ }^{\mathrm{b}} d$ denotes Cohen's measure of effect size. Degrees of freedom $=56$.

${ }^{*} \mathrm{p}<0.05$ (1-tailed; adjusted for multiple tests using the sequential Bonferroni procedure).

\section{Data Analyses}

An intention-to-treat design was incorporated where missing data associated with program dropout were imputed using the baseline carried forward method [24]. Because research suggests that short-term weight loss will usually revert to baseline soon after terminating treatment [25], this method, previously used under conditions similar to this study [26, 27], was selected. That is, when BMI data were missing at month 6 , the same participant's BMI score at baseline was imputed $[26,27]$. This method had the advantage of maximizing statistical power while not overestimating treatment effects by retaining data from only those with the highest treatment compliance, or estimating changes in BMI from participants who carried on complying [24]. Other missing data were imputed using the expectation maximization approach, which replaces each missing score with a set of plausible scores that represent the uncertainty about the correct score to impute. Discriminant procedures based on complete data sets from other subjects are then incorporated, and results are combined [28, 29]. This method is useful when response data are missing on a random basis $[28,29]$.

Dependent $t$ tests were first calculated for within-group changes in scores of tension, depression, total mood disturbance and BMI, associated with the 6 months of treatment. For effect size estimations, Cohen's $d$ was reported where 0.20 is considered a small effect, 0.50 is considered a moderate effect, and 0.80 is considered a large effect [30]. Pearson product moment correlations were then conducted between each mood measure at baseline and BMI change as well as between changes in each mood measure and BMI change. Partial correlations controlling for the baseline values of tension, depression, and total mood disturbance were also calculated. Correlations were then calculated between exercise session attendance and changes in each mood measure over 6 months. Finally, the correlation between exercise session attendance and change in BMI was derived. A correlation of 0.10 is considered small, 0.30 is considered moderate, and 0.50 is considered large [30].

Statistical significance was set at $\alpha=0.05$ (1-tailed) for the dependent $\mathrm{t}$ tests and $\alpha=0.05$ (2-tailed) for the correlation analyses. The sequential Bonferroni procedure suggested by Jaccard [31] and Holm [32] was used to adjust $\alpha$ levels for multiple tests. Thus, for the smallest $\mathrm{p}$ value (within a group of tests), the $\alpha$ value of 0.05 was divided by the number of tests conducted to establish the critical value for statistical significance (e.g. $0.05 / 4$ tests $=$ critical value of 0.01 ). If significance was met, the second smallest $\mathrm{p}$ value had the $0.05 \alpha$ value divided by the number of tests minus 1 , to establish the critical value (e.g. $0.05 / 3=$ critical value of 0.02 ). This process continued one-by-one with the progressively greater $\mathrm{p}$ values (e.g. 0.05 next divided by the number of tests minus 2 , or a critical value of 0.03 ) until statistical significance was no longer met. Research suggests
Table 2. Pearson product moment correlations between depression, tension, and total mood disturbance scores, and BMI change, over 6 months $(\mathrm{n}=57)$

BMI change, $r^{a}$

\section{Mood factors at baseline}

Depression $\quad 0.18$

Tension $\quad 0.12$

Total mood disturbance $\quad 0.25$

Changes in mood factors over 6 months

Depression change

Total mood disturbance change $\quad 0.33^{*}$

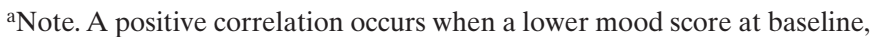
or greater reduction in score change from baseline to Month 6, is associated with a greater reduction in BMI.

$* \mathrm{p}<0.05$ (2-tailed; adjusted for multiple tests using the sequential Bonferroni procedure).

that this method performs better than the traditional Bonferroni correction procedure in maintaining statistical power, while also controlling for inflation of the type I error rate [33].

\section{Results}

Dependent $\mathrm{t}$ tests indicated statistically significant withingroup improvements over 6 months in scores of depression, tension, total mood disturbance, and BMI (table 1). The correlations between baseline scores on each mood measure and BMI change did not reach statistical significance (table 2). Correlations between changes over 6 months in depression and total mood disturbance and BMI change were significant (table 2). The correlation between change in tension and BMI change did not, however, reach statistical significance. Controlling for the respective depression, tension, and total mood disturbance scores at baseline did not strengthen correlations $(r=0.36,0.05$, and 0.22$)$.

The mean exercise session attendance percentage for the sample was $46.01 \pm 30.87$. Correlations between attendance percentage and changes in scores of tension $(r=-0.26)$ and total mood disturbance $(r=-0.32)$ were significant. The correlation between exercise session attendance and change in depression score approached statistical significance $(r=-0.21 ; \mathrm{p}=0.06)$. The correlation between exercise session attendance and change in BMI was significant $(r=-0.49)$.

\section{Discussion}

Significant within-group improvements in each mood measure were associated with the supported physical activity treatment. Through a social cognitive framework [16, 17], it could be interpreted that participation in the exercise pro- 
gram yielded feelings of self-efficacy and mastery that were associated with an enhanced psychological profile and better mood. Effect sizes for improvements in each of the mood factors were lower, however, than in studies of less severely obese women $[20,34]$ and in meta-analyses of results from the general adult population [9]. Although a dose-response relationship between exercise amounts and mood improvements was not found in most studies $[9,35]$, it has been suggested in others [36]. The exercise session attendance found here $(46 \%)$, which was lower than in samples of obese women of lower BMI (68\% [18] and 68\% [20]), thus could possibly have been a factor in the lower effect sizes observed in this sample. It should be noted that a significant association between exercise session attendance and mood changes were found, even with the comparatively low attendance rate. This suggested that the possible covariates to mood improvements just mentioned (self-efficacy and mastery) might have been proportional to one's commitment to the physical activity program (even if, overall, this commitment was quite limited). Direct testing for a threshold of frequency, duration, and intensity of physical activity for reliable changes in mood will add clarification.

Findings generally supported relations of reductions in negative mood from baseline to month 6 with reductions in BMI. This is consistent with previous research on women with a lower mean BMI [18]. Through social cognitive theory [16,17] and specifically the interpretation offered by Baker and Brownell [15], one may interpret this to mean that positive changes in psychological well-being may have led to improved internal resources to sustain a commitment to weight loss behaviors. As could be expected, exercise session attendance, a likely covariate of both caloric expenditure and a commitment to self-controlled eating [15], explained a significant $24 \%$ of the variance in BMI change.

A number of limitations of the present research should be noted. The self-select nature of participation may have included a disproportionate number of women with a propensity to exercise or who have mood affected by exercise. A symptom of depression, for example, is a lack of drive and volition, which may impede both accessing and following through with a physical activity program that may otherwise prove helpful with symptoms. As with all field investigations, it is possible that expectation effects or a desire to please study administrators affected results. Additionally, the small sample size and high attrition rate suggests that findings should be interpreted with caution.

Future research should incorporate greater statistical power and comprehensively investigate theoretical models of interrelations of physical activity, mood, eating behavior, and weight change. Constructs from social cognitive theory such as self-efficacy, self-image, and ability to utilize self-regulatory strategies may also prove to be fruitful additions for analyses. Additionally, further research is required to determine how episodes of negative affect that may cue overeating are related to the more stable measures of mood as analyzed here. The possibility that exercise affects psychological factors that are associated with overeating will also require more direct analyses, especially to determine if and how psychological changes in mood play a mediating role between physical activity and weight reduction through effects on disordered eating. Tests for moderating effects based on severity of overweight are also required, as are gender effects.

Considerable replication and extension will be required to better understand interrelations of physical activity, mood, eating, and weight loss across genders, obesity levels, ethnic groups, and in association with specific comorbidities (e.g. diabetes, heart disease). Incorporation of biochemical, satiety, and genetic factors may make explanatory models of weight loss more comprehensive. Continued related research is warranted to improve both weight loss theory and the treatment of obesity.

\section{Acknowledgement}

This research was supported by a grant from Kaiser Permanente.

\section{References}

1 Ogden CL, Carroll MD, Curtin LR, McDowell MA, Tabak CJ, Flegal KM: Prevalence of overweight and obesity in the United States, 1999-2004. JAMA 2006;295:1549-1555.

2 Aronne LJ: Classification of obesity and assessment of obesity-related health risks. Obes Res 2002;10(suppl 2):105S-115S.

3 Mann T, Tomiyama J, Westling E, Lew AM, Samuels B, Chatman J: Medicare's search for effective obesity treatments: diets are not the answer. Am Psychol 2007;62:220-233.

4 Santry HP, Gillen DL, Lauderdale DS: Trends in bariatric surgical procedures. JAMA 2005;294: 1909-1917.

5 Catenacci VA, Wyatt HR: The role of physical activity in producing and maintaining weight loss. Nat Clin Pract Endocrinol Metab 2007;3:518-529.
6 Miller WC, Koceja DM, Hamilton EJ: A metaanalysis of the past 25 years of weight loss research using diet, exercise, or diet plus exercise intervention. Int J Obes 1997;21:941-947.

7 Klem ML, Wing RR, McGuire MT, Seagle HM, Hill JO: A descriptive study of individuals successful at long-term maintenance of substantial weight loss. Am J Clin Nutr 1997;66:239-246.

8 Buckworth J, Dishman RK: Exercise Psychology. Champaign, Human Kinetics, 2002

9 Landers DM, Arent SM: Physical activity and mental health; in Singer RN, Hausenblas HA, Janelle CM (eds): Handbook of Research on Sport Psychology, 2nd ed. New York, Wiley, 2001, pp 740765 .
10 Blair SN, Connelly JC: How much physical activity should we do? The case for moderate amounts and intensities of physical activity. Res Q Exerc Sport 1996;67:193-205.

11 McElroy SL, Kotwal R, Malhotra S, Nelson EB, Keck PE, Nemeroff CB: Are mood disorders and obesity related? A review for the mental health professional. J Clin Psychiatry 2004;65:634-651.

12 van der Merwe MT: Psychological correlates of obesity in women. Int J Obes 2007;31(suppl 2): S31-S32.

13 Smith DE, Marcus MD, Lewis CE, Fitzgibbon M, Schreiner P: Prevalence of binge eating disorder, obesity, and depression in a biracial cohort of young adults. Ann Beh Med 1998;20:227-232. 
14 Toker S, Shirom A, Melamed S: Depression and the metabolic syndrome: gender-dependent associations. Depress Anxiety 2007; DOI: 10.1002/da 20379.

15 Baker CW, Brownell KD: Physical activity and maintenance of weight loss: physiological and psychological mechanisms; in Bouchard C (ed): Physical Activity and Obesity. Champaign, Human Kinetics:, 2000, pp 311-328.

16 Bandura A: Social Foundations of Thought and Action: A Social Cognitive Theory. Englewood Cliffs, Prentice Hall, 1986.

17 Bandura A: Self-Efficacy: The Exercise of Control New York, Freeman, 1997

18 Annesi JJ, Unruh JL: Relations of exercise, self-appraisal and mood changes, and weight loss in obese women: testing propositions based on Baker and Brownell's (2000) model. Am J Med Sci 2008;335: 198-204 (in press)

19 Annesi JJ: Effects of a cognitive behavioral treatment package on exercise attendance and drop out in fitness centers. Eur J Sport Sci 2003;3/2:1-12.

20 Annesi JJ: Effects of minimal exercise and cognitive behavior modification on adherence, emotion change, self-image, and physical change in obese women. Percept Mot Skills 2000;91:322-336.

21 McNair DM, Lorr M, Droppleman LF: Manual for the Profile of Mood States. San Diego, Education and Industrial Testing Service, 1992.
22 McAuley E, Peña MM, Jerome GJ: Self-efficacy as a determinant and an outcome of exercise; in Roberts G (ed): Advances in Motivation in Sport and Exercise. Champaign, Human Kinetics, 2001, pp 235-261.

23 Kaiser Permanente Health Education Services: Cultivating Health Weight Management Resource Guide. Portland, Kaiser Permanente, 2002.

24 Gadbury GL, Coffey CS, Allison DB: Modern statistical methods for handling missing repeated measurements in obesity trial data: beyond LOCF. Obes Rev 2003;4:175-184.

25 Jeffery RW, Drewnowski A, Epstein LH, Stunkard AJ, Wilson GT, Wing RR, Hill DR: Long-term maintenance of weight loss: current status. Health Psychol 2000;19(suppl):5-16.

26 Womble LG, Wadden TA, McGuckin BG, Sargent SL, Rothman RA, Krauthamer-Ewing ES: A randomized controlled trial of a commercial weight loss program. Obes Res 2004;12:1011-1018.

27 Teixiera PJ, Palmeira AL, Branco TL, Martins SS, Minderico CS, Brata JT, Silva AM, Sardinha LB: Who will lose weight? A reexamination of predictors of weight loss in women. Int J Behav Nutr Physical Activity 2004;1:12. www.ijbnpa.org/content/1/1/12.

28 Schafer JL: Multiple imputation: a primer. Stat Method Med Res 1999;8:3-15.
29 Rubin DB: Multiple Imputation for Nonresponse in Surveys. New York, Wiley, 1987.

30 Cohen J: Statistical Power for the Behavioral Sciences, 2nd ed. Hillsdale, Erlbaum, 1988.

31 Jaccard J: Interaction Effects in Factorial Analysis of Variance. Sage University Paper Series on Quantitative Applications in the Social Sciences, series no. 07-118. Thousand Oaks, Sage, 1998.

32 Holm D: A simple sequentially rejective multiple test procedure. Scand J Stat 1979;6:65-70.

33 Kromery JD, Dickson WB: The use of an overall $F$ test to control Type I error rates in factoral analysis of variance: limitations and better strategies. J Appl Behav Anal 1995;31:51-64.

34 Annesi, JJ, Unruh JL: Correlates of mood changes in obese women initiating a moderate exercise and nutrition information program. Psychol Rep 2006; 99:225-229.

35 King AC, Taylor CB, Haskell WL: Effects of differing intensities and formats of exercise training in psychological outcomes in older adults. Health Psychol 1993;12:292-300.

36 Dunn AL, Trivedi MH, Kampert JB, Clark CG, Chambliss HO: Exercise treatment for depression: efficacy and dose response. Am J Prev Med 2005; $28: 1-8$. 